\title{
Effect of Differences in the Exercise Frequency of Young People on Abdominal Strength and Muscle Thickness
}

\author{
Takanori Noguchi ${ }^{1, *}$, Shinichi Demura ${ }^{2}$ \\ ${ }^{1}$ Department of Industrial Business and Engineering, Fukui University of Technology, Fukui, Japan \\ ${ }^{2}$ Graduate School of Natural Science \& Technology, Kanazawa University, Kanazawa, Japan \\ *Corresponding author: t-noguchi@fukui-ut.ac.jp
}

Received October 28, 2014; Revised November 07, 2014; Accepted December 11, 2014

\begin{abstract}
Differences in the frequency of exercise among individuals increase during adolescence. These individual differences are associated with developmental differences in abdominal muscle groups that are closely related to activities of daily living. We examined the effect of differences in exercise frequency in young people on abdominal strength and muscle thickness. The subjects were 20 young male university athletes who belonged to sports clubs and who exercised $>6$ days per week for $>3$ h per day (athlete group: age, $20.1 \pm 1.43$ years; height, $171.7 \pm 6.74 \mathrm{~cm}$; weight, $67.9 \pm 10.41 \mathrm{~kg}$ ) and 20 young university male students who did not habitually exercise (less than twice per week) (nonathlete group: age, $20.1 \pm 1.41$ years; height, $171.6 \pm 5.46 \mathrm{~cm}$; weight, $63.2 \pm 8.62$ $\mathrm{kg}$ ). Their physical characteristics (height, weight, and body mass index), abdominal flexion strength, and abdominal muscle thickness (rectus abdominis, external oblique, internal oblique muscles) were measured. Although no significant differences were found in the physical characteristics of either group, abdominal strength and all muscle thickness were significantly greater in the athlete group than that in the nonathlete group. In addition, a relatively high correlation between abdominal strength and muscle thickness was found only in the athlete group $(r=0.73)$. In conclusion, abdominal strength and muscle thickness were greater in young athletes who frequently exercise compared with that in nonathletes. Abdominal muscle strength increased with increasing abdominal muscle thickness in the athletes but not in the nonathletes.
\end{abstract}

\section{Keywords: development of muscle function, ultrasound imaging, adolescence}

Cite This Article: Takanori Noguchi, and Shinichi Demura, "Effect of Differences in the Exercise Frequency of Young People on Abdominal Strength and Muscle Thickness." American Journal of Sports Science and Medicine, vol. 2, no. 6 (2014): 218-221. doi: 10.12691/ajssm-2-6-3.

\section{Introduction}

Physical fitness develops with age from infancy, and after reaching a peak at approximately 20 years, it begins to gradually decrease. Hence it is very important to sufficiently increase the physical fitness level during adolescence because it affects physical fitness during subsequent years [1]. In addition, development of muscle function peaks at approximately 20 years of age [2]. Above all, abdominal muscle groups contribute to enhancing intraabdominal pressure, stabilizing the vertebral column, maintaining posture, and movements such as before-backward flexion, twisting, and sideward flexion of the trunk [3]. In addition, increasing abdominal strength is important for enabling smooth movements. This is because such movements are needed for activities of daily living such as sitting up, ascending and descending stairs, and rising from a chair. It has been reported that a decrease in physiological functions, including muscle strength, is associated with the frequency and content of exercise $[4,5,6]$. In addition, decreases in muscle strength lead to further reductions in the frequency of physical activity. Hence it is important to sufficiently enhance muscle strength during adolescence.
Individual differences in exercise frequency in young people are very large. In Japan, until high school, sports and physical activities are mainly performed in physical education class and club activities in schools [7]. However, after graduating from high school, differences in the frequency and content of exercise increase among individuals, and very few students belong to athletic clubs even after enrolling into colleges or universities. We examined the effect of differences in exercise frequency in young people on abdominal strength and muscle thickness and the relationship between these two parameters.

\section{Methods}

\subsection{Subjects}

The subjects were 20 young male university athletes who belonged to sports clubs (baseball, $n=5$; swimming, $\mathrm{n}=3$; boxing, $\mathrm{n}=1$; canoe, $\mathrm{n}=3$; tennis, $\mathrm{n}=4$; soft tennis, $\mathrm{n}=4$ ) and exercised $>6$ days per week for $>3$ h per day (athlete group: age, $20.1 \pm 1.43$ years; height, $171.7 \pm 6.74$ $\mathrm{cm}$; weight, $67.9 \pm 10.41 \mathrm{~kg}$ ) and 20 young university male students who exercised less than twice per week (nonathlete group: age, $20.1 \pm 1.41$ years; height, $171.6 \pm$ 
$5.46 \mathrm{~cm}$; weight, $63.2 \pm 8.62 \mathrm{~kg}$ ). All subjects were healthy and did not have any physical problems. Before the experiment, the purpose and procedures were explained in detail and written informed consent was obtained from all participants. The experimental protocol was approved by the Ethics Committee on Human Experimentation of the Faculty of Human Science, Kanazawa University (No. 2012-14).

\subsection{Procedures}

In this study, abdominal flexion strength and the thickness of each abdominal muscle were measured. The measurement devices and procedures used are described below.

\subsubsection{Abdominal Flexion Strength Test}

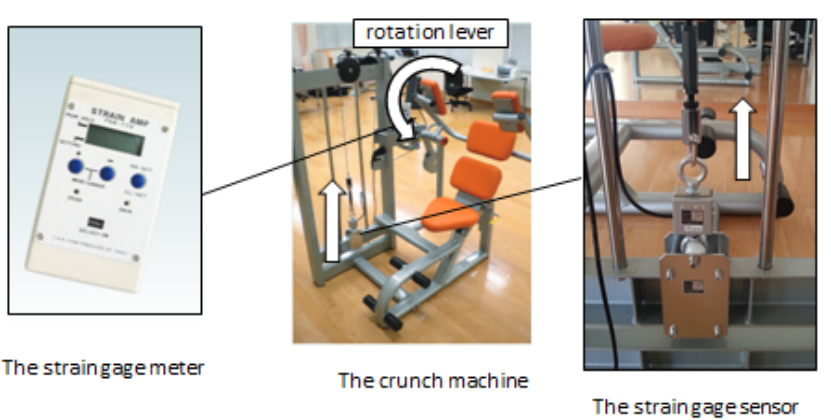

Figure 1. The trunk strength measurement device

The strain gage sensor

- range: $0-100 \mathrm{~kg}$

- measurement error: under $\pm 0.5 \%$
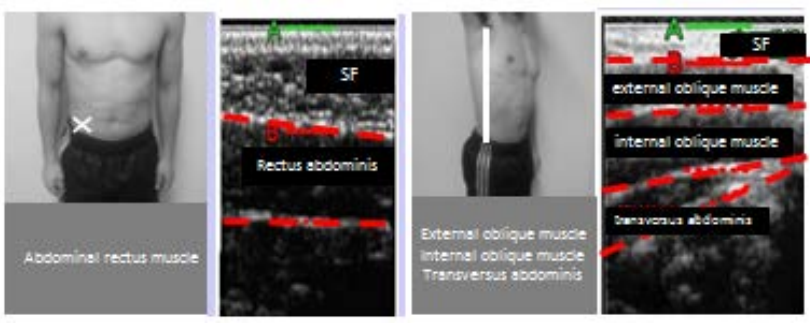

Figure 2. Measurement sites of the ultrasound imaging device

Left: rectus abdominis

right: external obliquemuscle, internal oblique muscle, transversusabdominus

SF: subcutaneous fat

A trunk strength measurement device (original model; Takei Scientific Instruments Co. Ltd., Tokyo, Japan) was used to measure the static strength of abdominal flexion. This device was newly developed to measure isometric strength during forward bending of the trunk $[15,16]$. When pushing the rotation lever at chest height, the exerted strength was measured through a strain gauge sensor (range: 0-100 kg, measurement error: under \pm $0.5 \%$ ) connected through a pulley (Figure 1). After adjusting the chair height to match the seated height of each subject, the subjects sat so as to match their abdominal flexion point on the rotation axis of the lever. After placing both elbows on the base of the rotation lever with arms folded, the subjects maximally exerted abdominal flexion strength with their timing. At that time, the tester checked to see if the subject be able to push the lever with their hands or straighten their back.

The value measured through a pulley was revised according to the following formula:

$$
\begin{aligned}
& \text { Abdominal flexion strength } \\
& =\text { measurement value }(\mathbf{k g}) \times \mathbf{0 . 6}
\end{aligned}
$$

After one practice trial, the test was performed twice with 1-min rest between each trial to eliminate fatigue.

\subsubsection{Thickness of Abdominal Muscles}

An ultrasound imaging device (GT-101; TANITA, Tokyo, Japan) was used to measure the thickness of abdominal muscles. Figure 2 shows computer-displayed ultrasound images acquired with the B-mode method. The thickness of three muscles (rectus abdominis, external oblique muscle, internal oblique muscle) was measured using a probe frequency of $6 \mathrm{MHz}$. The thickness of the rectus abdominis was measured as the maximal width of a point 4-cm transverse from the navel, excluding tendinous intersections. The thickness of the rectus abdominis was measured as the maximal width of a point 4-cm transverse from the navel, excluding tendinous intersections. Because the other two parts have overlapping organizations, they were measured at the same position [8,9]. In other words, we measured two thirds of a line drawn horizontally from the height of the navel perpendicular to a vertical axillary line (Figure 2). All measurements were completed by a single experienced tester. The thickness of each abdominal muscle was measured twice in an upright standing position with muscles tensed to increase abdominal pressure.

\subsection{Statistical Analyses}

Intra-class correlation coefficients (ICCs) were calculated to evaluate the trial-to-trial reliabilities of abdominal flexion strength and the thickness of each abdominal muscle. The mean differences in age, physique characteristics [height, weight, body mass index (BMI)], abdominal strength, and muscle thickness between the athlete and the nonathlete groups were examined by using an unpaired t-test. The mean difference was assessed by effect size (ES). The relationships between abdominal flexion strength and muscle thickness were examined by determining the Pearson's correlation coefficients. Statistical significance $(\alpha)$ was set at $p<0.05$, which was adjusted by using the Bonferroni method.

\section{Results}

The trial-to-trial reliabilities of abdominal flexion strength and the thickness of each abdominal muscle had very high ICCs of $>0.93$. Table 1 shows the results for the mean differences in age, physical characteristics, abdominal flexion strength, and muscle thicknesses and their ES values between the two groups. No significant differences were found for age, height, weight, and BMI between the groups. On the other hand, abdominal flexion strength and thickness of all muscles were significantly greater in the athlete group (ES = 1.38-2.85). 
Figure 3 shows the correlations between abdominal flexion strength and the total of the abdominal muscle thickness. A significant and high correlation was found only in the athlete group ( $\mathrm{r}=0.73, \mathrm{p}<0.05)$.

Table 1. Means differences of age, physique characteristics, abdominal flexion strength and muscle thicknesses between the athlete group and the non-athlete group

\begin{tabular}{|c|c|c|c|c|c|c|c|c|c|c|}
\hline & & & \multicolumn{2}{|c|}{ Athlete group $(n=20)$} & \multicolumn{2}{|c|}{ Kon-athlete gruop $(\mathrm{n}=20)$} & \multirow[b]{2}{*}{ difference } & \multirow[b]{2}{*}{$\mathrm{t}$} & \multirow[b]{2}{*}{$\mathrm{P}$} & \multirow[b]{2}{*}{ ES } \\
\hline & & & Mean & SD & Mean & $\mathrm{SD}$ & & & & \\
\hline \multirow{4}{*}{$\begin{array}{c}\text { physique } \\
\text { characteristics }\end{array}$} & age & (yr) & 20.1 & 1.43 & 20.1 & 1.41 & 0.05 & 0.11 & 0.91 & 0.04 \\
\hline & height & $(\mathrm{cm})$ & 171.7 & 6.74 & 171.6 & 5.46 & 0.16 & 0.08 & 0.93 & 0.03 \\
\hline & weight & (kg) & 67.9 & 10.41 & 63.2 & 8.62 & 0.50 & 1.61 & 0.12 & 026 \\
\hline & BMI & & 23.0 & 2.90 & 21.4 & 2.39 & 0.59 & 1.91 & 0.07 & 0.59 \\
\hline strength & abdominal strength & $(\mathrm{kg})$ & 46.2 & 11.59 & 21.9 & 3.27 & 24.29 & $10.55 *$ & 0.00 & 2.85 \\
\hline \multirow{3}{*}{ thickness } & rectus abdominis & $(\mathrm{mm})$ & 17.5 & 3.48 & 12.6 & 1.82 & 4.97 & $5.99 *$ & 0.00 & 1.79 \\
\hline & external oblique muscle & $(\mathrm{mm})$ & 8.0 & 1.93 & 5.7 & 1.28 & 2.26 & $4.47 *$ & 0.00 & 138 \\
\hline & internal oblique muscle & $(\mathrm{mm})$ & 15.8 & 2.84 & 10.8 & 2.23 & 4.97 & $6.69 *$ & 0.00 & 1.95 \\
\hline
\end{tabular}

$*: \mathrm{p}<0.05 / 3=0.0167$

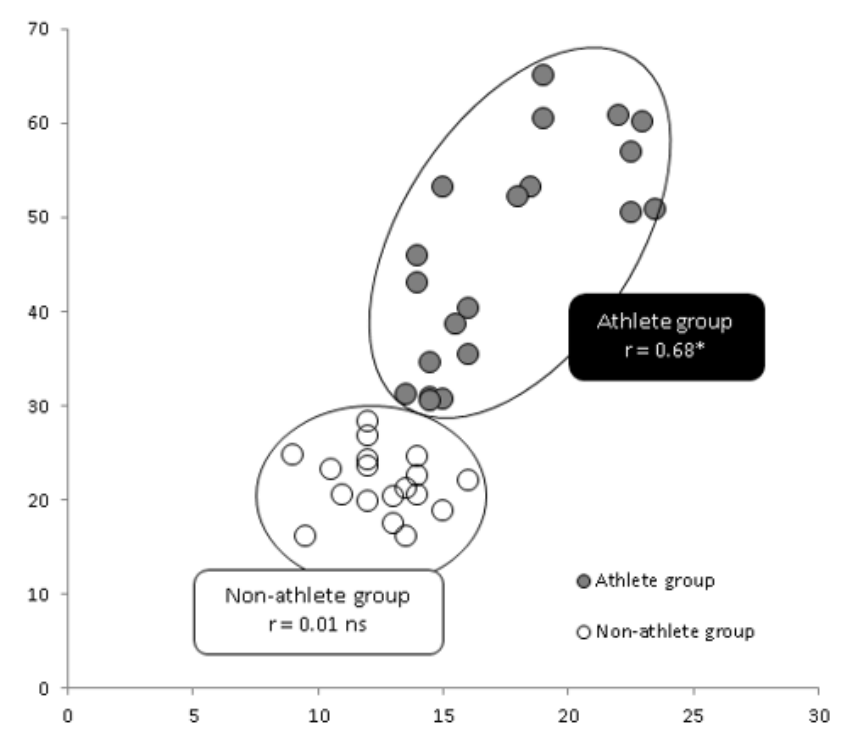

Figure 3. Correlations between abdominal flexion strength and the total of abdominal muscle strength

\section{Discussion}

Although muscle strength develop until adolescence, it gradually decreases with age until it reaches a level required for activities of daily living in old age [10,11] unless persons do not intentionally exercise. Young persons who increased strength to a higher level in adolescence had greater margins of strength in middle and old age, and were better able to avoid succumbing to serious situations, such as becoming bedridden, compared with young persons who did not increase their strength during adolescence [2].

In addition, if muscle strength is sufficiently increased in adolescence, persons can be more physically active in later stages of life, which helps prevent marked decreases in strength in old age. In short, sufficient muscle strength will produce good circulation of an exercise cycle would be generated. In this study, we focused on the abdominal muscle groups because they are important in various physical activities, including fundamental activities of daily living, and we also examined the effect of exercise frequency on abdominal strength, and muscle thickness in university students.

We showed that the athletes in this study had better developed abdominal strength and thicker abdominal muscle groups than did the nonathletes. The athletes belonged to sports clubs and had trained for several hours almost every day, so they were a group with a very high exercise frequency. It is inferred that in addition to the strength of the limbs and trunk required in competitive sports, abdominal strength also developed. In contrast, nonathletes had thickness of all abdominal muscle groups (rectus abdominis, external oblique, internal oblique) and abdominal strength inferior to those of the athletes, despite having no differences in physical characteristics and age. Notably, there was a large difference in the abdominal strength (approximately $15 \mathrm{~kg}$ ) between the two subject groups. The rectus abdominis, external oblique, and internal oblique muscles are surface layer muscle groups associated with large force exertion among the abdominal muscle groups [3]. Hypo functionality of abdominal muscle groups is linked to a decrease in physical activities and posture changes and is a cause of lumbar pain [12,13]. Therefore, there is a high probability that having inferior strength as a young person can largely affect activities of daily living in the future. Consequently, it may be important for young people in general to enhance muscle strength in adolescence.

Increased muscle thickness was associated with a proportionate increase in strength in the athlete group but not in the nonathlete group. In general, muscle thickness is also related to muscle volume [14], and thicker muscle groups are associated with increased strength [7,9]. Differences in strength development between the two groups may also be because of differences in the rate of mobilization per motor unit, which may be related to nerve impulses [15]. Because the nonathlete group had a lower exercise frequency or insufficient exercise experience in the past, their neuromuscular connections may have been more poorly developed. It is possible that neural impulses were not sufficiently transmitted to muscles and the mobilization rate of voluntary muscles was low when these subjects attempted to exert muscle strength. It is difficult to develop abdominal strength because the stimulation of general activities of daily living may be insufficient for developing strength. To develop abdominal strength, young people who do not frequently exercise should increase their exercise frequency, perform physical activities regularly, and adopt a routine exercise program for strength development.

A limitation of this study is that only males were selected as subjects in this study. Young females generally have predominantly inferior muscle development compared with that of young males. Therefore, young females may show results different from those of young males. The effect of differences in the exercise frequency 
of young females on abdominal strength and muscle thickness should be investigated in a future study.

In conclusion, because of their higher exercise frequency, the athletes in this study had more highly developed abdominal strength and thicker abdominal muscles compared with the nonathletes. In general, young persons with low exercise frequency of the same age have lower abdominal strength and thickness of the rectus abdominis, external oblique, and internal oblique muscles relative to those of athletes. In this study a correlation between abdominal flexion strength and the total of the abdominal muscle thickness was observed in the athletes but not in in the nonathletes, which suggested that there is a relationship between abdominal strength and abdominal muscle thickness. So abdominal muscle training is important for the nonathletes, and they need it.

\section{Acknowledgement}

This work was supported by the Japan Society for the Promotion of Science (JSPS) KAKENHI Grant-in-Aid for Young Scientists (B) Number 24700673. The authors would like to thank Enago (www.enago.jp) for the English language review.

\section{References}

[1] Barnekow-Bergkvist, M., Hedberg, G., Janlert, U. and Jansson, E., "Prediction of physical fitness and physical activity level in adulthood by physical performance and physical activity in adolescence--an 18-year follow-up study," Scandinavian journal of medicine \& science in sports, 8 (5 Pt 1). 299-308. Oct. 1998.

[2] Demura, S., Health and a Sports Science Lecture the 2nd Edition, Tokyo, Japan. Kyorinsyoin, 2011, 105-125. [Japanese]

[3] Duchateau, J., "Bed rest induces neural and contractile adaptations in triceps surae,” Medicine and science in sports and exercise, 27 (12). 1581-1589. Dec. 1995.

[4] El Ouaaid, Z., Shirazi-Adl, A., Plamondon, A. and Larivière, C., "Trunk strength, muscle activity and spinal loads in maximum isometric flexion and extension exertions: a combined in vivocomputational study,” Journal of Biomechanics, 46 (13). 22282235. Sep. 2013.
[5] Fleg, J.L. and Lakatta, E.G., "Role of muscle loss in the age associated reduction in VO2 max," Journal of applied physiology, 65 (3). 1147-1151. Sep. 1988.

[6] Ferrari, A.U., Radaelli, A. and Centola, M., "Aging and the cardiovascular system,” Journal of Applied Physiology, 95 (6). 2591-2597. 2003.

[7] Ferreira, P.H., Ferreira, M.L. and Hodges, P.W., "Changes in recruitment of the abdominal muscles in people with low back pain: ultrasound measurement of muscle activity,” Spine, 29 (22). 2560-2566. Nov. 2004.

[8] Huang, Y.C. and Malina, R.M., "Physical activity and healthrelated physical fitness in Taiwanese adolescents," Journal of physiological anthropology and applied human science, 21 (1). 11-9. Jan. 2002.

[9] Hunter, S.K., Thompson, M.W. and Adams, R.D., "Relationships among age-associated strength changes and physical activity level, limb dominance, and muscle group in women," The journals of gerontology. Series A, Biological sciences and medical sciences, 55 (6) A. B264-B273. Jun. 2000.

[10] Izquierdo, M., Aguado, X., Gonzalez, R., López, J.L. and Häkkinen, K., "Maximal and explosive force production capacity and balance performance in men of different ages," European journal of applied physiology and occupational physiology, 79: 260-267. Feb. 1999.

[11] Macaluso, A. and De Vito, G., "Muscle strength, power and adaptations to resistance training in older people," European journal of applied physiology, 91 (4). 450-472. Apr. 2004.

[12] Mikkelsson, L., Kaprio, J., Kautiainen, H., Kujala, U., Mikkelsson, M. and Nupponen, H., "School fitness tests as predictors of adult health-related fitness," American Journal of Human Biology, 18 (3). 342-349. May-Jun. 2006.

[13] Nahhas Rodacki, C.L., Luiz Felix Rodacki, A., Ugrinowitsch, C., Zielinski, D. and Budal da Costa, R., "Spinal unloading after abdominal exercises,” Clinical biomechanics, 23 (1). 8-14. Jan. 2008.

[14] Naka, H. and Demura, S., "Influence of habitual exercise on physique and physical fitness in adolescent male students: From an examination of three-year longitudinal data,” Japanese Journal of Physical Education, 39. 288-304. 1994.

[15] Noguchi, T., Demura, S., Shimada, S., Kobayashi, H., Yamaji, S. and Yamada, T., "Effect of Sports Club Activities on the Physique and Physical Fitness of Young Japanese Males,” World Journal of Education, 3 (6). 27-32. 2013a.

[16] Noguchi, T., Demura, S. and Takahashi, K., "Relationships between Sit-Ups and Abdominal Flexion Strength Tests and the Thickness of Each Abdominal Muscle," Advances in Physical Education, 3 (2). 84-88. May. 2013b.

[17] Yamamoto, T., For Practical and Scientific Conditioning Measurement and Assessment, Book House HD, 2004, 18-19. 